\title{
Simulation of gyrotrons using the high-order particle-in-cell code PICLas
}

\author{
$\underline{\text { S. M. Copplestone }}{ }^{1}$, P. Ortwein ${ }^{1}$, C.-D. Munz ${ }^{1}$, K. A. Avramidis ${ }^{2}$ and J. Jelonnek ${ }^{2}$ \\ ${ }^{1}$ Institute of Aerodynamics and Gas Dynamics, University of Stuttgart, Stuttgart, Germany, copplestone@iag.uni-stuttgart.de \\ ${ }^{2}$ Institute for Pulsed Power and Microwave Technologies, Karlsruhe Institute of Technology, Karlsruhe, Germany
}

Maxwell-Vlasov modelling of gyrotron devices offer deep insight into the relevant physics at nowadays moderate computational costs. The particle-incell (PIC) method with its long history [1,2] is a prominent example of kinetic methods for tackling such problems. Advantages of high-order Discontinuous Galerkin Methods [3] in combination with PIC schemes have since been developed [4] and are applied within the state-of-the-art code PICLas [5]. Recent improvements within this framework include high-order geometry approximation using curvilinear body-fitted instead of piecewise linear meshes and non-conforming interfaces with hanging nodes. Accurate PIC simulations of gyrotrons with open boundaries additionally require sophisticated modelling in the vicinity of open boundary conditions in order to prevent spurious reflections from corrupting the solution within the physical domain [6]. The simulation of a test-case involving a model $140 \mathrm{GHz} \mathrm{TE}_{0,3}$ gyrotron [7] with and without the occurrence of dynamic after cavity interaction (ACI) is investigated regarding numerical settings and the identification of resonant dynamic ACI modes in the tapered part of the gyrotron geometry is shown via dynamic mode decomposition (DMD) [8]. It is shown that an inappropriate choice of specific parameters can lead to unfavourable results. For a more realistic application, a $140 \mathrm{GHz}$ $\mathrm{TE}_{28,8}$ gyrotron is simulated and the results are discussed.

Full-wave PIC simulations of gyrotron devices are conducted using PICLas, a code which combines high-order spatial and temporal discretisations by applying the discontinuous Galerkin spectral element approach in combination with curvilinear boundary handling. In PICLas, Maxwell's equations in purely hyperbolic form [9] are solved and the hyperbolic correction factor is set to unity throughout this work. Possible sources of numerical reflection at open boundaries, when particles or electromagnetic waves interact with the domain boundaries, are accommodated by absorbing layers, so-called perfectly matched layers (PML) [6].

The academic test-case of a model $140 \mathrm{GHz} \mathrm{TE}_{0,3}$ gyrotron with two different external magnetic field profiles with either a constant or a spatially varying distribution is considered. For details on the parameters of the setup see [7]. Depending on the applied magnetic field, the occurrence of dynamic ACI is encountered on which, additionally, the applied boundary conditions take effect. For the PIC setup of the model gyrotron, a hexahedral curvilinear mesh with a geometrical order $\mathrm{O}(5)$ containing 2080 cells is used for a total length of the model of $120 \mathrm{~mm}$. The boundary conditions at the entry and exit of the gyrotron are modelled open (first order absorbing) and the surface of the gyrotron tubing via a perfectly electric conducting (PEC) condition. The spatial and temporal order are $\mathrm{O}(6)$ and $\mathrm{O}(4)$, respectively. Time integration is done explicitly with a low storage Runge-Kutta scheme. The current beam is modelled by 85000 particles with a weighting factor of $10^{5}$. Particle-grid coupling is carried out by using a polynomial shape function [4] and the spatial spread of each particle can be adjusted. The simulations are performed on $60 \mathrm{CPU}$, which uses $\approx 50$ CPUh for 1 ns simulation time. The output power reaches $9.2 \mathrm{~kW}$ (averaged over several cycles) and is calculated by integration of the Poynting vector over a cross section of the gyrotron at 120 $\mathrm{mm}$. PMLs with one extra layer of cells are installed at the entry and exit planes of the gyrotron. The additional cell layers either continue the taper angle of the outer radius in order to prevent a kink in the geometry or they have a cylindrical shape with a constant radius. The results are post-processed by means of DMD, for details on the algorithm see [11], for which 563 complete 3D solutions of the electric field are used, from 109.1 ns to 109.923381 ns with a sampling time-step of $0.2381 \mathrm{ps}$. The frequencies and growth rates found are shown in Fig. 1 for a non-uniform (case A) and uniform (case B) external magnetic field $\mathrm{B}_{\mathrm{z}}=5.561 \mathrm{~T}$ with a cylindrical PML region where the damping is set to zero (small damping values yield no significant differences). The DMD reveals frequencies $\omega_{\mathrm{i}}$ and growth rates $\omega_{\mathrm{r}}$ of the fundamental mode at $\approx$ $140 \mathrm{GHz}$ and its harmonics.

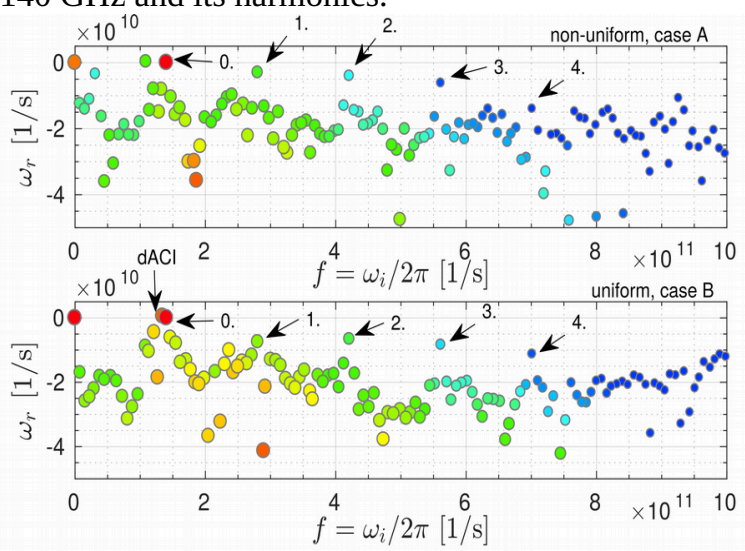

Fig. 1. DMD of the electric field: Frequencies and growth rates for the non-uniform (case A, top) and uniform (case B, bottom) external magnetic field. Note that the colour and size of the dots correspond to the Euclidean norm, correlating with the energy content, of each mode.

In case A, the fundamental frequency at 140.30 $\mathrm{GHz}$ is visible and no dynamic ACI is encountered. Note that the mode visible at $109 \mathrm{GHz}$ does not represent any physically relevant structures. Case B shows two distinct frequencies near $140 \mathrm{GHz}$, the fundamental frequency at $140.30 \mathrm{GHz}$ and the dynamic 
ACI at $134.34 \mathrm{GHz}$. Additionally, the mode pattern of the DMD data of case B is depicted in Fig. 2 for the fundamental and dynamic ACI frequencies. The position in the tapered part of the gyrotron, where the dynamic ACI is excited and propagated along the gyrotron axis is clearly visible. Both the fundamental and dynamic ACI modes show a $\mathrm{TE}_{0,3}$ structure.

$$
\text { dmd(Etheta) / a.u. }
$$

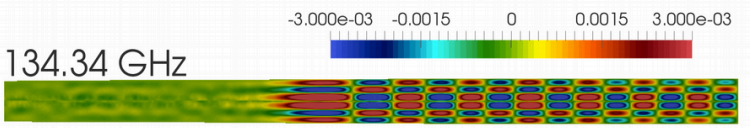

\section{$140.30 \mathrm{GHz}$}

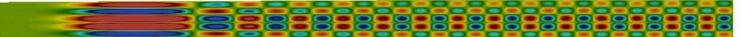

Fig. 2. DMD of the electric field in the axial $x-y-p l a n e$ of the gyrotron showing the resonant fundamental (bottom) and dynamic ACI mode (top) for case B (cylindrical PML).

To investigate the influence of reflection at open boundaries, a parameter variation of the damping factor $\zeta$ and PML outer radius tapering is conducted. The results are summarized in the table below. For low damping factors $\left(\zeta=5 \cdot 10^{9}\right)$ and a tapered PML, the dynamic ACI frequency and output power slightly change as compared to the cylindrical PML geometry with no or small damping factors. This changes for larger damping values $\left(\zeta=50 \cdot 10^{9}\right)$, where the presence of a dynamic ACI is not observed and the frequency of the fundamental mode slightly decreases. For very high damping factors $\left(\zeta=500 \cdot 10^{9}, \zeta=5000 \cdot 10^{9}\right)$, the dynamic ACI frequency reduces by up to $\approx 2 \mathrm{GHz}$ and for a cylindrical PML a shift from $\mathrm{TE}_{0,3}$ to $\mathrm{TE}_{2,3}$ structure is observed for the dynamic ACI mode. This may be linked to the heavy change in reflection properties at the exit plane of the gyrotron, which changes the backward scattering and excitation of certain modes in the uptaper of the gyrotron.

PML damping factor 3 , DMD frequency f, mode pattern and output Power for different cases. Note that all results refer to a uniform external magnetic field $\mathrm{B}_{\mathrm{z}}$.

\begin{tabular}{|c|c|c|c|c|c|}
\hline $\begin{array}{c}3 \\
{\left[10^{9}\right]}\end{array}$ & $\mathrm{f}$ [GHz] & $\begin{array}{c}\text { TE } \\
\text { Mode }\end{array}$ & $\begin{array}{c}\mathrm{P}_{\text {out }} \\
{[\mathrm{kW}]}\end{array}$ & $\begin{array}{l}\text { PML } \\
\text { radius }\end{array}$ & $\overline{A C I}$ \\
\hline \multirow{2}{*}{5} & 140.253 & 0,3 & \multirow{2}{*}{9.4} & \multirow{2}{*}{ tapered } & \multirow{2}{*}{ yes } \\
\hline & 134.036 & 0,3 & & & \\
\hline 50 & 140.035 & 0,3 & 9.7 & tapered & no \\
\hline \multirow{2}{*}{500} & 140.366 & 0,3 & \multirow{2}{*}{10.4} & \multirow{2}{*}{ tapered } & \multirow{2}{*}{ yes } \\
\hline & 133.473 & 0,3 & & & \\
\hline \multirow{2}{*}{5000} & 140.30 & 0,3 & \multirow{2}{*}{10.0} & \multirow{2}{*}{ const. } & \multirow{2}{*}{ yes } \\
\hline & 132.31 & 3,2 & & & \\
\hline
\end{tabular}

In general, the results agree very well with findings reported in [7] for low and moderate damping factors in the PML. However, for larger values, we find increasing differences. As shown in [6], too high damping values attenuate the electromagnetic waves inside the PML too fast, hence, increasing the numerical reflection because the PML then functions comparable to a PEC surface, in which no fields exist due to the infinite conduction of the material.

The physical as well as the PIC parameters of the $\mathrm{TE}_{28,8}$ gyrotron are chosen according to the case presented in [10] with a slightly increased length of the geometry due to two additional cell layers of PML regions and the entry and exit. The mesh has a length of
$121 \mathrm{~mm}$ in $\mathrm{z}$-direction, the largest diameter being $43.9 \mathrm{~mm}$, and consists of 3472 hexahedral curvilinear mesh cells of geometrical order $\mathrm{O}(7)$. The spatial and temporal order of are $\mathrm{O}(10)$ and $\mathrm{O}(4)$, respectively. During stationary operation, there are $\approx 400000$ particles with a weighting factor of $10^{6}$. The external magnetic field of $5.587 \mathrm{~T}$ and electric current of $44 \mathrm{~A}$ of the electron hollow beam for soft excitation operation are used. At this point, the hard excitation parameters are used in order to shift into operation mode of the gyrotron. For this setup we observe the stable operation of $\mathrm{TE}_{27,8}$ mode, which is in constrast to [10]. We also conducted different simulations with different grids and resolutions and observed the same result. We link these observations to the different numerical model with less reflection at the input and output planes due to the PML regions.

\section{References}

1. C. K. Birdsall and A. B. Langdon. Plasma Physics via Computer Simulation. Hilger, 1991.

2. R. W. Hockney and J. W. Eastwood. Computer Simulation Using Particles. Taylor \& Francis, 1988.

3. J. S. Hesthaven and T. Warburton. Nodal Discontinuous Galerkin Methods: Algorithms, Analysis, and Applications. Texts in Applied Mathematics. Springer, 2008.

4. G. B. Jacobs and J. S. Hesthaven. High-order Nodal Discontinuous Galerkin Particle-in-cell Method on Unstructured Grids. In: J. Comput. Phys. 214.1 (2006), pp. 96-121.

5. C.-D. Munz et al. Coupled Particle-In-Cell and Direct Simulation Monte Carlo method for simulating reactive plasma flows. Comptes Rendus Mecanique 342.1011 (2014), pp. 662-670.

6. S. M. Copplestone et al. Complex-Frequency Shifted PMLs for Maxwell's Equations With Hyperbolic Divergence Cleaning and Their Application in Particle-inCell Codes. In: IEEE Transactions on Plasma Science 45.1 (2017), pp. 2-14.

7. K. A. Avramidis et al. A comparative study on the modeling of dynamic after-cavity interaction in gyrotrons. In: Physics of Plasmas 22.5 (2015), p. 053106.

8. P. J. Schmid. Dynamic mode decomposition of numerical and experimental data, Journal of Fluid Mechanics 656 (2010), pp. 5-28.

9. C.-D. Munz et al. A three-dimensional finite-volume solver for the Maxwell equations with divergence cleaning on unstructured meshes. Computer Physics Communications, 130.1-2 (2000), pp. 83-117.

10. A. Stock. A High-Order Particle-in-Cell Method for Low Density Plasma Flow and the Simulation of Gyrotron Resonator Devices. PhD thesis, University of Stuttgart, 2013.

11. O. Schmidt. Numerical investigations of instability and transition in streamwise corner-flows. $\mathrm{PhD}$ thesis, University of Stuttgart, 2014. 